\title{
Intramolecular Energy Transfer in a Bichromophoric System, Zinc meso-Tetratolylporphyrin Covalently Linked to Anthracene through Ethylene Linkage
}

\author{
Eun Ju Shin \\ Department of Chemistry, Sunchon National University, Sunchon, Chonnam 540-742, Korea. E-mail: ejs@sunchon.ac.kr \\ Received January 24, 2006
}

Key Words : Porphyrin, Anthracene, Fluorescence, Intramolecular energy transfer

Porphyrins ${ }^{1-13}$ have been received great attention as suitable photoactive components for the study of energy and electron transfer reactions in multicomponent systems and as building blocks for molecular photonic wires, ${ }^{4,5}$ molecular gates, ${ }^{6}$ chemosensors, ${ }^{7}$ and other molecular electronic and photonic devices, because of their attractive electronic and redox properties and with the ease of modification by the development of various synthetic routes. The fact that there are many sites on the porphyrin at which to attach electron-releasing or electron-withdrawing groups suggests that tremendous electronic modulation of the porphyrin should be possible. ${ }^{8}$

The photoinduced energy or electron transfer processes in multicomponent donor-acceptor systems including porphyrin derivatives have been extensively studied as model systems for primary charge separation in natural photosynthesis. ${ }^{1,2}$ Many of these studies involve bichromophoric systems called as dyad, in which porphyrin chromophore is linked covalently to some electron/energy donor or acceptor chromophore. Such bichromophoric systems provide the possibility that one of the chromophores may act as an antenna to absorb light in a spectral region where the other does not absorb, but which may then transfer this energy to the other chromophore by electronic energy transfer or electron transfer.

Based on the relative excited state energies and other photophysical properties, both covalently bonded and selfassembled dyad molecules involving anthracene as a donor and porphyrin as an acceptor have been found to be particularly attractive candidates for studying energy migration and the development of electronic and photonic devices.

We report the preparation and spectroscopic properties of a dyad (ZnPor-E-An) involving zinc porphyrin covalently linked by ethylene spacer to anthracene and its intramolecular energy transfer has been discussed.

Figure 1 shows the absorption spectrum of ZnPor-E-An in dichloromethane along with those of two isolated components: ZnPor-I and 9-vinylanthracene in Scheme 1. Absorption spectrum of ZnPor-E-An shows characteristic zinc porphyrin spectrum with a strong Soret (B) band at $420 \mathrm{~nm}$ and two weak visible $Q$ bands at 549 and $588 \mathrm{~nm}$ (see Table 1). The sum of the absorption spectra of two components ZnPor-I and 9-vinylanthracene exactly matched the absorption spectrum of ZnPor-E-An, indicating that there is no electronic interaction between porphyrin and anthracene

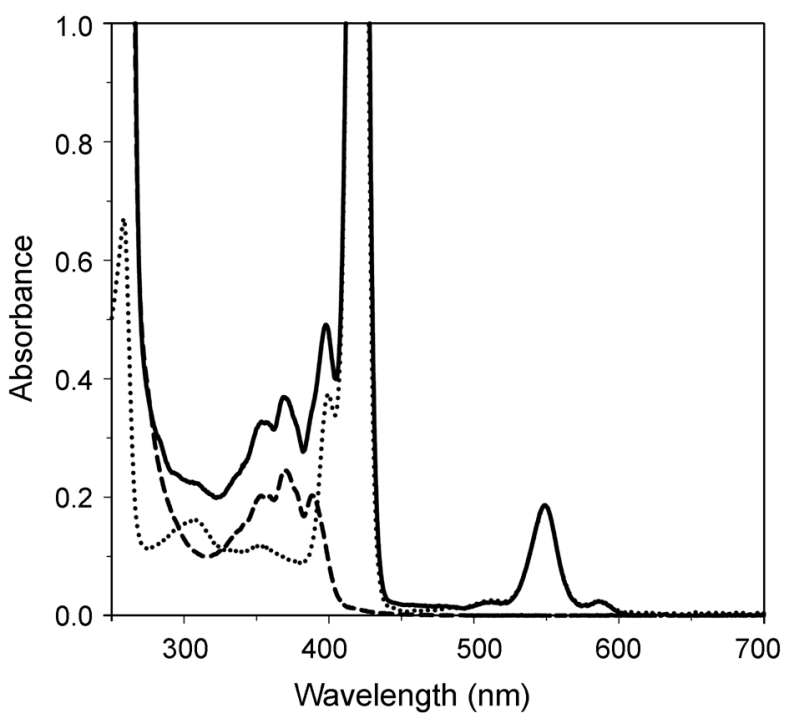

Figure 1. Absorption spectra of ZnPor-E-An (solid line), ZnPor-I (dotted line), and 9-vinylanthracene (dashed line) in dichloromethane.

moieties in the ground state. Ethylene was employed here as a spacer group between porphyrin and anthracene.

Figure 2 presents the fluorescence spectra of ZnPor-E-An in dichloromethane along with those of two isolated components: ZnPor-I and 9-vinylanthracene. The fluorescence spectrum of ZnPor-E-An with excitation at $550 \mathrm{~nm}$, corresponding to the absorption region of the porphyrin moiety, consists of two bands with maxima at 593 and $645 \mathrm{~nm}$, showing characteristic fluorescence of zinc porphyrin, although the ratios of two band intensities at 593 and $645 \mathrm{~nm}$ are different and fluorescence quantum yield of ZnPor-E-An is similar to that of $\mathrm{ZnPor}-\mathrm{I}$.

However, when excitation of ZnPor-E-An is made at $370 \mathrm{~nm}$, in the region where the anthracene chromophore absorbs, fluorescence emission appears with two maxima at 593 and $645 \mathrm{~nm}$, along with fluorescence band around $435 \mathrm{~nm}$ corresponding to the fluorescence region from the anthracene moiety in Figure 2. Although not perfectly matched, the emission of ZnPor-E-An overlaps with the sum of the emission from $\mathrm{ZnPor}$ and anthracene moieties. This means that there is not significant electronic interaction between porphyrin and anthracene moieties.

On excitation in the anthracene absorption region, the 

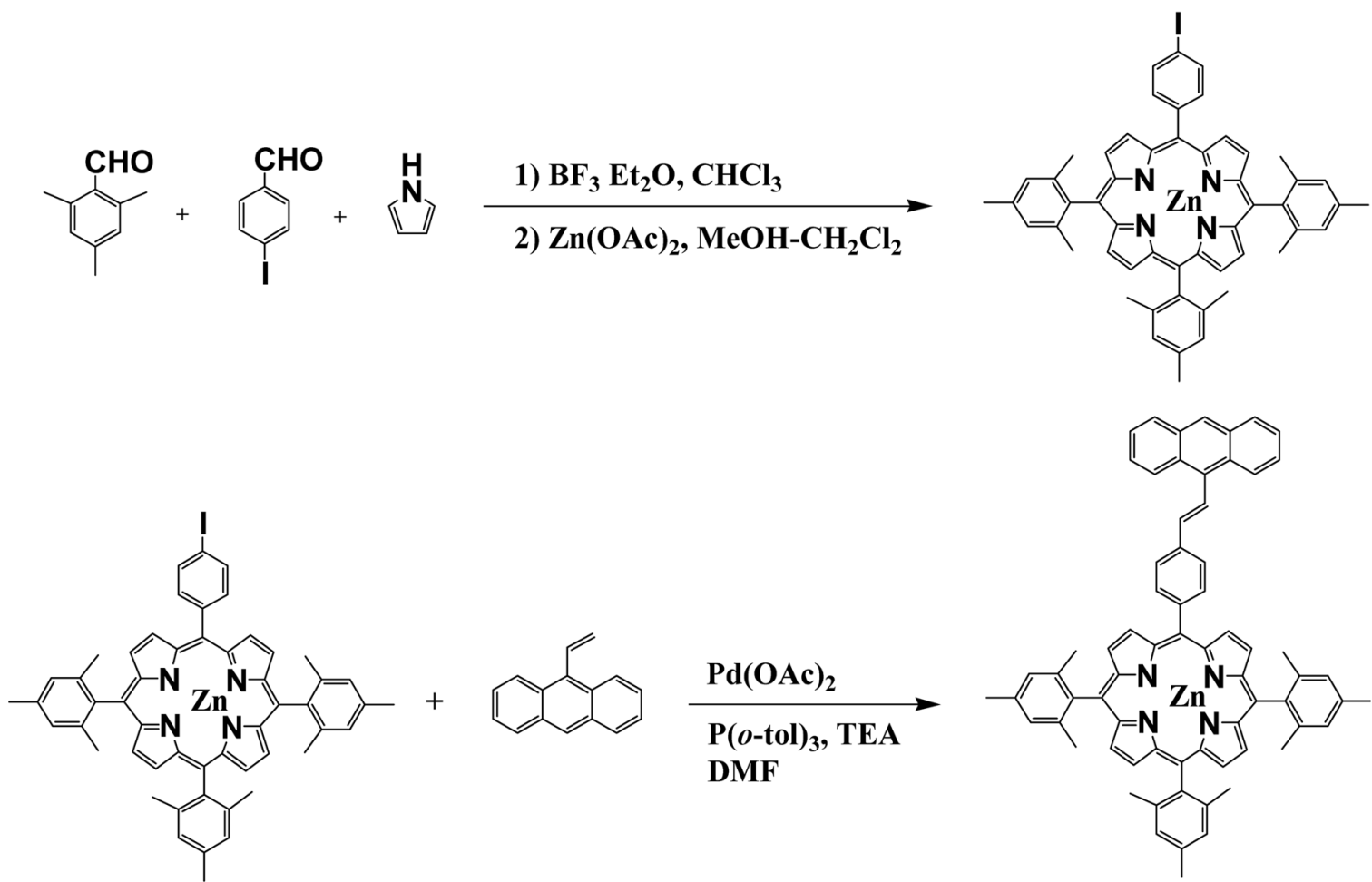

ZnPor-I

9-vinylanthracene

ZnPor-E-An

Scheme 1. Synthesis of ZnPor-E-An.

Table 1. Absorption and fluorescence data of ZnPor-E-An in $\mathrm{CH}_{2} \mathrm{Cl}_{2}$

\begin{tabular}{|c|c|c|c|c|c|c|}
\hline \multirow{2}{*}{ compound } & \multicolumn{3}{|c|}{$\lambda_{\mathrm{a}}^{\max }(\mathrm{nm})$} & \multirow{2}{*}{$\begin{array}{l}\lambda_{\mathrm{f}}^{\max } \\
(\mathrm{nm})\end{array}$} & \multirow{2}{*}{$\Phi_{\mathrm{f}}$} & \multirow{2}{*}{ remark } \\
\hline & - & Soret & Q-band & & & \\
\hline \multirow{2}{*}{ ZnPor-E-An } & \multirow{2}{*}{$308,369,398$} & \multirow{2}{*}{420} & \multirow{2}{*}{549,587} & 593,645 & 0.014 & excitation at $550 \mathrm{~nm}$ \\
\hline & & & & $407,435,593,645$ & 0.08 & excitation at $370 \mathrm{~nm}$ \\
\hline ZnPor-I & 308,400 & 420 & 549,586 & 593,645 & 0.013 & excitation at $550 \mathrm{~nm}$ \\
\hline 9-vinyl-anthracene & $358,370,388$ & - & - & 412,430 & $0.76^{\mathrm{a}}$ & excitation at $370 \mathrm{~nm}$ \\
\hline
\end{tabular}

${ }^{a}$ data from ref. 13

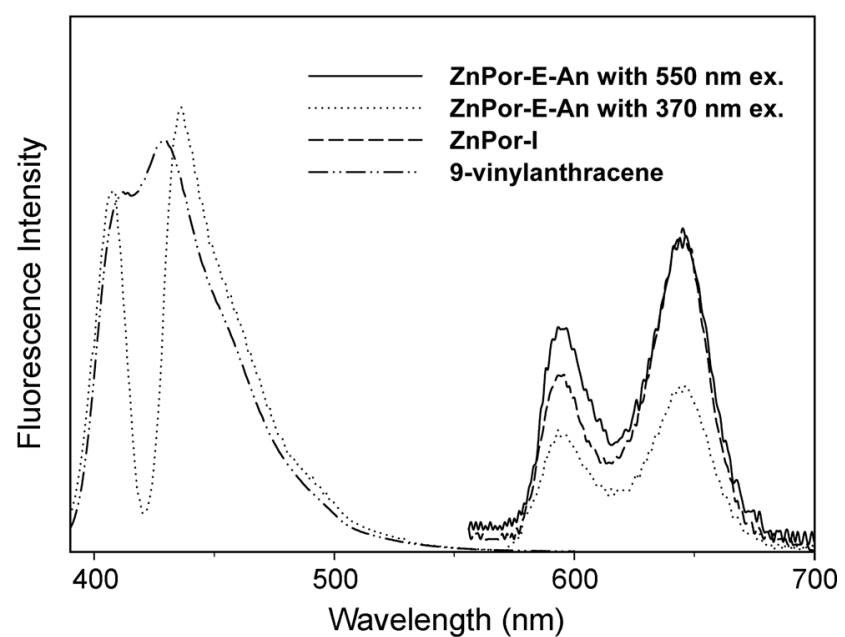

Figure 2. Fluorescence spectra of ZnPor-E-An (solid line: with excitation at $550 \mathrm{~nm}$, dotted line: with excitation at $370 \mathrm{~nm}$ ), ZnPor-I (dashed line), and 9-vinylanthracene (dash-dotted line) in dichloromethane. emission from two moieties of ZnPor and 9-vinylanthracene has been observed (Figure 2), implying the singlet-singlet energy transfer from anthracene to porphyrin moiety. The evidence that these are not the emission arising from direct excitation of both chromophores comes from the fluorescence excitation spectrum with $\lambda_{\mathrm{em}}=645 \mathrm{~nm}$ (Figure 3). The observation that the fluorescence excitation spectrum with $\lambda_{\mathrm{em}}=645 \mathrm{~nm}$ (the emission region from ZnPor moiety) is similar to the absorption spectrum of ZnPor-E-An reveals that emission of $645 \mathrm{~nm}$ comes not only from the direct excitation of ZnPor moiety, but also from the singlet-singlet energy transfer from anthracene to ZnPor moiety. The efficiency of energy transfer to ZnPor moiety should not be $100 \%$ on excitation in the anthracene band. The distance and orientation of donor and acceptor groups in anthraceneporphyrin energy transfer in dyad system may possibly be important here.

Both intra and intermolecular energy transfer may be found to occur with ZnPor-E-An. The use of dilute solution 


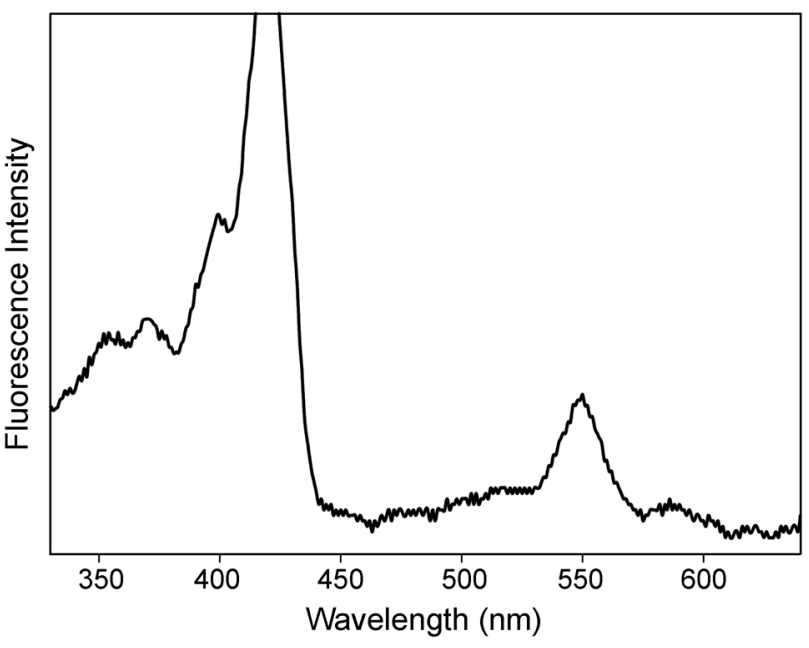

Figure 3. Fluorescence excitation spectrum of ZnPor-E-An with emission at $645 \mathrm{~nm}$ in dichloromethane.

makes it very unlikely that there is any contribution from intermolecular energy transfer, such that only intramolecular processes occur.

Fluorescence quantum yields were obtained in dichloromethane for ZnPor-E-An and the model compound ZnPor-I, with excitation at $550 \mathrm{~nm}$, where the anthracene does not absorb. As shown in Table 1, the similar values were obtained to be 0.014 and 0.013 for ZnPor-E-An and ZnPor-I, respectively. Fluorescence quantum yield in dichloromethane for ZnPor-E-An with excitation at $370 \mathrm{~nm}$ in the anthracene absorption region is 0.08 . Considering the area ratio of the fluorescence only from ZnPor moiety (570-680 nm) and total fluorescence, fluorescence quantum yield is roughly estimated to be about 0.007 . Comparing this value $(0.007)$ with fluorescence quantum yield (0.014) of ZnPor-E-An with excitation at $550 \mathrm{~nm}$, maximum energy transfer efficiency can be calculated to be $50 \%$, assuming that the singlet-singlet energy transfer from anthracene to ZnPor moiety is the sole reason for fluorescence from $\mathrm{ZnPor}$ moiety.

In summary, ZnPor-E-An dyad consisting zinc porphyrin and anthracene has been prepared. In this ZnPor-E-An dyad, ethylene was used as spacer group between zinc porphyrin and anthracene moieties. Absorption and fluorescence spectra indicate that there is no electronic interaction between zinc porphyrin and anthracene moieties. On excitation at the region of anthracene absorption, the observation of emission from zinc porphyrin moiety as well as anthracene moiety should imply the singlet energy transfer from anthracene to zinc porphyrin moiety.

\section{Experimental Section}

Synthesis of 5-(4-iodophenyl)-10,15,20-tris(2,4,6-trimethylphenyl)porphyrin (Por-I). 5-(4-iodophenyl)-10,15, 20-tris(2,4,6-trimethylphenyl)porphyrin (Por-I) was prepared according to Linsey procedure ${ }^{14}$ reported for closely related tetraarylporphyrin systems. To $0.58 \mathrm{~g}(2.5 \mathrm{mmol})$ of 4-iodobenzaldehyde and $1.11 \mathrm{~mL}(7.5 \mathrm{mmol})$ of 2,4,6trimethylbenzaldehyde in $1 \mathrm{~L}$ of chloroform at room temperature was added $0.70 \mathrm{~mL}(10 \mathrm{mmol})$ of pyrrole and $0.38 \mathrm{~mL}$ $(3 \mathrm{mmol})$ of $\mathrm{BF}_{3} \cdot \mathrm{O}(\mathrm{Et})_{2}$. The mixture was stirred for $1 \mathrm{hr}$ and then $1.70 \mathrm{~g}(7.5 \mathrm{mmol})$ of 2,3-dichloro-5,6-dicyano-1,4benzoquinone was added. The reaction mixture was stirred for $1 \mathrm{hr}$ and evaporated and dried under vacuum. Column chromatography on silica gel with dichloromethane $/ n$-hexane (2/1) gave $0.33 \mathrm{~g}$ of Por-I in $c a .15 \%$ yield. ${ }^{1} \mathrm{H}$ NMR in $\mathrm{CDCl}_{3}$ (400 MHz): $\delta 8.54-8.68(8 \mathrm{H}, \mathrm{m}$, pyrrole), $7.99(2 \mathrm{H}$, $\mathrm{d}, J=8.0 \mathrm{~Hz}$, 4-iodophenyl), $7.84(2 \mathrm{H}, \mathrm{d}, J=8.0 \mathrm{~Hz}$, iodophenyl), $7.18(6 \mathrm{H}, \mathrm{m}, 2,4,6$-trimethylphenyl), $2.54(9 \mathrm{H}$, s, para-Ar-methyl), 1.77 (6H, s, ortho-15-Ar-methyl), 1.76 (12H, s, ortho-10\&20-Ar-methyl), -2.77 (2H, s, pyrroleNH). FAB-MS: $[M+\mathrm{H}]^{+} m / z 868$.

Synthesis of zinc 5-(4-iodophenyl)-10,15,20-tris(2,4,6trimethylphenyl)porphyrin (ZnPor-I). A $1: 4$ methanol : dichloromethane solution of Por-I with an excess of zinc acetate was stirred at room temperature for $24 \mathrm{hrs}$. The solution was chromatographed on a short silica gel column with the same solvent mixture to remove excess zinc acetate and the solvent was evaporated. Dark-purple solid was obtained. The reaction progress was monitored by change of absorption and fluorescence spectra.

Synthesis of zinc 5-(4-(2-(9-anthryl)ethenyl)phenyl)10,15,20-tris(2,4,6-trimethylphenyl)porphyrin (ZnPor-EAn). $23 \mathrm{mg}(0.14 \mathrm{mmol})$ of 9-vinylanthracene and $2.3 \mathrm{mg}$ $(0.01 \mathrm{mmol})$ of palladium acetate, $11.9 \mathrm{mg}(0.04 \mathrm{mmol})$ of tri-ortho-tolylphosphine, and $30 \mathrm{mg}(0.3 \mathrm{mmol})$ of triethylamine were added to a solution of $112 \mathrm{mg}(0.12 \mathrm{mmol})$ of ZnPor-I in $10 \mathrm{~mL}$ dry dimethylformamide, at room temperature. The reaction mixture was stirred at $100{ }^{\circ} \mathrm{C}$ for $5 \mathrm{~h}$, and then cooled to room temperature. $50 \mathrm{~mL}$ dichloromethane was added and the mixture was washed with aqueous $\mathrm{NH}_{4} \mathrm{Cl}$ and dried with sodium sulfate and then evaporated under vacuum. The final work-up involved chromatography on silica using dichloromethane/ $n$-hexane $(1 / 3)$ as eluent and crystallization from dichloromethane/ $n$-hexane, giving 14 $\mathrm{mg}\left(12 \%\right.$ yield) red-orange crystals. ${ }^{1} \mathrm{H} \mathrm{NMR}$ in $\mathrm{CDCl}_{3}(300$ $\mathrm{MHz}): \delta 8.64-8.80(8 \mathrm{H}, \mathrm{m}$, pyrrole $), 8.33(1 \mathrm{H}, \mathrm{d}, J=16 \mathrm{~Hz}$, ethylene), 8.23-8.25 (2H, m, anthracene 1,8-H), $7.99(2 \mathrm{H}, \mathrm{d}$, $J=8.0 \mathrm{~Hz}, 5$-phenyl), 7.91-7.93 (2H, m, anthracene 4,5-H), $7.84(2 \mathrm{H}, \mathrm{d}, J=8.0 \mathrm{~Hz}, 5-$ phenyl), 7.63-7.64 (2H, m, anthracene 2,3-H), 7.44-7.46 (2H, m, anthracene 6,7-H), 7.37-7.41 (6H, m, 2,4,6-trimethylphenyl), $7.20(1 \mathrm{H}, \mathrm{s}$, anthracene $10-\mathrm{H}), 5.55(1 \mathrm{H}, \mathrm{d}, J=16 \mathrm{~Hz}$, ethylene), 2.55 (9H, s, para-Ar-methyl), 1.74 (18H, s, ortho-Ar-methyl). FAB-MS: $[M+\mathrm{H}]^{+} m / z 1008$.

Spectroscopic measurements. ${ }^{1} \mathrm{H}$ NMR spectra were measured on a $400 \mathrm{MHz}$ Bruker Avance 400 NMR spectrometer in chloroform- $d_{1}$. EI-Mass and FAB-Mass spectra were measured on Micromass (UK) platform II GC/LC Mass Spectrometer and Jeol LTD JMS-HX110/110A High Resolution Tandem Mass Spectrometer, respectively. Absorption spectra were recorded on a Shimadzu UV-2401PC spectrophotometer. Steady-state fluorescence spectra were recorded on a SLM-Aminco AB2 luminescence spectro- 
photometer. The concentrations were controlled to be $c a .1 \times$ $10^{-5} \mathrm{M}$, where the absorbances of the solutions at the excitation wavelength of $360 \mathrm{~nm}$ had usually the value of $0.07-0.08$, to avoid inner filter effects. Fluorescence spectra were corrected for the wavelength response of the system. Fluorescence quantum yields $\Phi_{\mathrm{f}}$ were determined using zinc 5,10,15,20-tetra(4-methylphenyl)porphyrin (ZnTTP) as a standard $\left(\Phi_{\mathrm{f}}=0.049\right.$ in dichloromethane $){ }^{15}$

Acknowledgements. The author would like to thank KBSI for their help in the measurement of NMR and mass spectra. This work was supported by a grant No. R01-2006000-10262-0 from the Basic Research Program of the Korea Science \& Engineering Foundation.

\section{References}

1. Wasielewski, M. R. Chem. Rev. 1992, 92, 435.

2. Gust, D.; Moore, T. A.; Moore, A. L. Acc. Chem. Res. 1993, 26, 198.

3. Kurreck, H.; Huber, M. Angew. Chem. Int. Ed. Engl. 1995, 34,
849

4. Martin, R. E.; Diederich, F. Angew. Chem. Int. Ed. Engl. 1999, 38, 1350

5. Wagner, R. W.; Lindsey, J. S. J. Am. Chem. Soc. 1994, 116, 9759.

6. Wagner, R. W.; Lindsey, J. S.; Seth, J.; Palaniappan, V.; Bocian, D. F. J. Am. Chem. Soc. 1996, 118, 3996.

7. Cho, H. S.; Song, J. K.; Ha, J.-H.; Cho, S.; Kim, D.; Yoshida, N.; Osuka, A. J. Phys. Chem. A 2003, 107, 1897.

8. Lindsey, J. S.; Prathapan, S.; Johnson, T. E.; Wagner, R. W. Tetrahedron 1994, 50, 8941.

9. Balzani, V.; Moggi, L.; Scandola, F. Supramolecular Photochemistry; Balzani, V. D., Ed.; Reidel: Dordrecht, 1987; pp 1-28.

10. Kalyanasundaram, K. Photochemistry of Polypyridine and Porphyrin Complexes; Academic Press: London, 1992.

11. Ahn, T. K.; Kim, K. S.; Kim, D. Y.; Noh, S. B.; Aratani, N.; Ikeda, C.; Osuka, A.; Kim, D. J. Am. Chem. Soc. 2006, 128(5), 1700 .

12. Hwang, I.-W.; Aratani, N.; Osuka, A.; Kim, D. Bull. Korean Chem. Soc. 2005, 26(1), 19.

13. Ko, D.; Kim, H.; Park, J. H.; Kim, D.; Sim, E. Bull. Korean Chem. Soc. 2005, 26(10), 1505.

14. Shin, E. J.; Kim, D. Bull. Korean Chem. Soc. 2003, 24, 1490.

15. Berlman, I. B. Handbook of Fluorescence Spectra of Aromatic Molecules, ${ }^{\text {nd }}$ Ed.; Academic Press: New York, 1971. 